\title{
Design and Manufacture of Scale-Model System of Highway Bridge Subjected to a Running Vehicle
}

\author{
Shuirong Gui \\ ${ }^{\text {a }}$ School of Transportation, \\ Southeast University, \\ Nanjing, china \\ ${ }^{\mathrm{b}}$ School of Civil Engineering and Architecture, \\ East China Jiao Tong University, \\ Nanchang, China \\ guishuirong@163.com
}

\author{
Shui Wan \\ School of Transportation, \\ Southeast University, \\ Nanjing, china \\ Shuisheng Chen \\ School of Civil Engineering and Architecture, \\ East China Jiao Tong University, \\ Nanchang, China
}

\begin{abstract}
According to vibration characteristics of highway bridge-vehicle coupling, taking a simple supported beam bridge as an example, the scale model test system of vehicle-bridge coupling vibration has been designed and manufactured, including model testing bridge, testing vehicle, traction, braking, limiting device and a data collecting system. Comparison of measured value and theoretical value, the dynamic characteristics of model bridge and testing car have tested efficiency of design and manufacture about model bridge and testing car; Comparison of scale model test results with numerical results of spring mass vibration system and simulation results of LS-DYNA, which verifies the similitude laws of bridgevehicle interaction model text and corrects the reliability of measurement result. The results show the feasibility of model test equipment and the validity of the test results.
\end{abstract}

Keywords-Highway bridge; vehicle-bridge coupling; scale test model; design and Manufacture;numerical verification

\section{INTRODUCTION}

Impact effect of bridge subjected to moving vehicle has been affected by many factors such as the dynamic characteristic of vehicle, the dynamic characteristic of bridge structure, vehicle speed, bridge deck roughness, the number of loading vehicle and the route of vehicle running. The varied factors make the research of vehicle-bridge coupling system becoming very complex [1]. Numerical analysis and field test have been used for resolving the problem of vehicle-bridge coupling vibration. The method of numerical analysis contains two ways, one is the equation of vehicle-bridge coupling vibration deduced accompanying the vehicle simplified as spring-mass-damp system refer to reference [2], the other is the 3-D refine finite element model of vehicle-bridge coupling system is established with LS-DYNA refer to reference [3]. At present, many scholars at home and abroad research the problem of vehicle-bridge coupling mainly focusing on the first method [4 7]. Due to the complexity of influence factors, the bridge model and vehicle model must be simplified to a large extent in solving the response of vehicle-bridge coupling using numerical simulation. Some scholars at home and abroad $[3,8]$ analyze the responses of vehicle-bridge coupling using LS-
DYNA to establish the 3-D refine finite element model. The method is hard to be put into use widely because of costing plenty of computation time and unable to consider the effect of road roughness on the response of vehicle-bridge coupling.

The reliability of measured data can be improved obviously in indoor model-test because the results can be rarely interfered in and the same loading condition can be implemented repeatedly. Many dynamic scale-model tests about bridge have been carried out, while most of them research the dynamic property of bridge structure [9], the dynamic response of bridge subjected to earthquake load $[10,11]$ and wind load and so on The simple scale-model test equipment of vehicle-bridge coupling has been made to identify the response of bridge subjected to moving vehicle in reference [12]. In Tommy's model test, the dynamic characteristics of vehicle and bridge haven't been considered. Based on the characteristics of vehicle-bridge coupling vibration, taking a simple supported beam bridge as an example, the scale-model test equipment of vehicle-bridge coupling vibration has been designed and manufactured according to the similarity law. The numerical responses of vehicle-bridge coupling have been resolved by means of spring-mass-damper system and refine finite element model of LS-DYNA. The feasibility of scale-model test equipment of vehicle-bridge coupling system has been verified through comparison of responses using three difference methods.

\section{Design And Manufacture ABout ScAle-Model TeSt}

\section{A. Similarity of Relation}

In order to ensure the similarity of physical mechanics, test model and prototype model must meet similarity relations as follow, similarity of physical dimension, similarity of material stress and strain, similarity of quality and gravity, similarity of boundary condition. The relation of scale each physical quantity is derived by dimension analysis and $\pi$ theorem when researching the response of vehicle-bridge coupling vibration in linear elastic range [13]. The variable $\lambda$ is defined as similarity ration. At the same time, the variable $\lambda_{l}, \lambda_{E}, \lambda_{\rho}$ represents geometric scale, elasticity modulus scale and density 
scale. $\lambda_{u}, \lambda_{F}, \lambda_{t}$ represents deformation scale, force scale and time scale. $\lambda_{v m}, \lambda_{v k}, \lambda_{v c}$ represents quality scale of vehicle, stiffness scale of vehicle and damp scale of vehicle.

Elasticity similitude law must keep the similarity relation between inertia force and elastic restoring force which is often used in researching natural vibration characteristic of structure. According to the demand of force similarity, the Eq.1 can be derived:

$$
\lambda_{t}^{2}=\lambda_{l}^{2} \cdot \lambda_{E}^{-1} \cdot \lambda_{\rho}
$$

The force similarity must be guaranteed in researching the dynamic response of structure during elasticity phase. According to the force similarity, the Eq. 2 can be derived as follow.

$$
\lambda_{F}=\lambda_{E} \cdot \lambda_{1} \cdot \lambda_{u}
$$

The gravity similarity requires the inertia force and gravity keep the same scale. According to the gravity similarity, the Eq.3 can be derived:

$$
\lambda_{t}=\lambda_{l}^{1 / 2}
$$

The elastic force and gravity similarity must satisfy elastic similarity and gravity similarity simultaneously:

$$
\lambda_{\rho}=\lambda_{E} \cdot \lambda_{l}^{-1}
$$

The similarity of dynamic characteristic can be satisfied through adding weight according to the material used in model test. The additional weight can be computed as the follow equation:

$$
m_{a d}=m_{p} /\left(\lambda_{E} \cdot \lambda_{l}^{2}\right)-m_{m}
$$

Where, $m_{p}$ represents quality of prototype structure, $m_{m}$ represents quality of model structure.

Similarity scale of model car dynamic characteristic can be derived in terms of dimensional analysis and external load similarity.

$$
\lambda_{v m}=\lambda_{l}^{3} \cdot \lambda_{\rho}, \quad \lambda_{v k}=\lambda_{l} \cdot \lambda_{E}, \quad \lambda_{v c}=\lambda_{E} \cdot \lambda_{l}^{1 / 2} \cdot \lambda_{u}
$$

The similarity law of vehicle-bridge coupling scale-model test sees literature [14].

\section{B. Design and Manufacture}

1) Introduction of model test

The $30 \mathrm{~m}$ simple supported beam bridge is chosen as the research object. The bridge is composed of five $\mathrm{T}$ beam with $2.0 \mathrm{~m}$ high. The five $\mathrm{T}$ beams are connected into a whole through the diaphragm plate and wet joint of flange plate. The bridge deck pavement is covered with $10 \mathrm{~cm}$ thickness asphalt concrete pavement and $8 \mathrm{~cm}$ thickness C50 concrete cast-inplace layer. The parameter of prototype vehicle is used in numerical simulation as shown in Table I, and the parameter of model car can be deduced according to Eq.6. The vibration response of vehicle-bridge coupling is researched when a vehicle running on the normal lane. The cross section of beam and loading location is shown in Fig.1.

TABLE I. VEHICLE PARAMETER

\begin{tabular}{|c|l|l|l|}
\hline name & $\begin{array}{c}\text { Mass } \\
\mathbf{( k g )}\end{array}$ & $\begin{array}{c}\text { Stiffness } \\
\mathbf{( N / m m )}\end{array}$ & $\begin{array}{c}\text { Distance with the } \\
\text { centre of mass of } \\
\text { vehicle body (m) }\end{array}$ \\
\hline Front wheel & 594 & 28000 & 3.4 \\
\hline Mid wheel & 932 & 33900 & 0.2 \\
\hline Rear wheel & 932 & 33900 & 1.4 \\
\hline Vehicle body & 30542 & $/$ & $/$ \\
\hline Front suspension & 6300 & 27.3 & \\
\hline Mid suspension & 7900 & 38.4 & \\
\hline Rear suspension & 7900 & 38.4 & \\
\hline
\end{tabular}

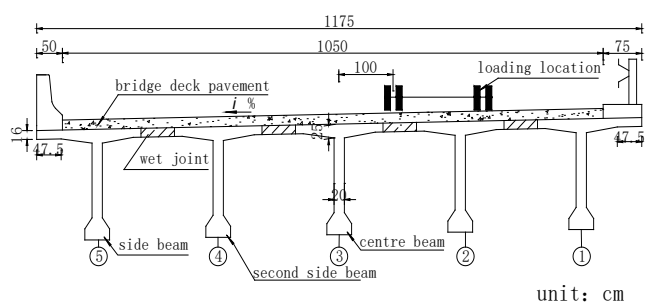

Fig. 1. Location of vehicle loads

The equipment of vehicle-bridge coupling scale-model is consisted of many components as follow, accelerating region of bridge approach, testing region of model-bridge, decelerating region of leaving bridge, equipment of traction and braking, the equipment of lateral limiting. Many factors such as geometric scale, material of model-bridge and boundary conditions of bridge must be taken into account in designing model testing bridge. Considering the length of vehicle accelerating and braking, laboratory room where can be used in testing, the geometrical scale $\lambda_{l}$ is chosen as 10 . According to $\lambda_{l}$, some parameters of model testing system are computed, testing region is $3 \mathrm{~m}$, accelerating region is $10 \mathrm{~m}$, braking region is $4 \mathrm{~m}$, and the whole model test system need $17 \mathrm{~m}$.The model testing bridge is made with $0.2 \mathrm{~m}$ high, $1.175 \mathrm{~m}$ width. The diagrammatic sketch of model test equipment about vehicle-bridge coupling system is shown in Fig.2.

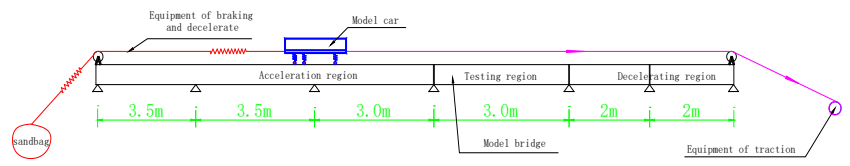

Fig. 2. Diagrammatic sketch of model test about vehicle-bridge coupling system

\section{2) Bridge model}

a)Model material

The purpose of the test and applicable scope of different material must be taken into account in selecting material of model-bridge. Metallic material, plastic, gypsum and micro- 
concrete are often used in model test. Gypsum is suitable for the dam body and foundation soil in model test and microconcrete is often used in strength model of reinforced concrete. Load scale $\lambda_{F}$ is direct proportion to elasticity modulus scale $\lambda_{E}$ according to Eq. 2. If elasticity modulus scale is enlarged, the load decreases largely. The external load is offered by test car in model test. The greater the quality of test car is, the more the inertia force increase following the length of accelerating, braking and decelerating increase obviously. Low elasticity modulus and high strength material is often chosen to make model test-bridge. Metallic material, gypsum and microconcrete can meet with the above-mentioned requirements. The PMMA is fragile with high strength and low elastic modulus in low temperature and low stress. PMMA is transparent, at the same time it can be made different shape and detecting flaw easily. Considering various factors, the PMMA is chosen as the material of model-bridge at end.

\section{b) Manufacture of model-bridge}

The model bridge is divided into five $\mathrm{T}$ beam which has been made solely, then the five $\mathrm{T}$ beam is glued into a whole with glass cement. Three different thickness of PMMA plate such as $20 \mathrm{~mm}, 16 \mathrm{~mm}$ and $8 \mathrm{~mm}$ are chosen based on requirement of model-bridge geometric size. During making the $\mathrm{T}$ beam, there are many principles must be complied by as follow. Firstly, variable thickness web is chosen according to the actual size. Secondly, it must ensure the girder vertical bending stiffness. Lastly, the bridge deck is equivalent to rectangular plate with uniform thickness. Longitudinal joint crack is staggered between the web, flange plate and horseshoe.

\section{c) Selecting constant of model test}

Five normal cuboids of PMMA are measured with vernier caliper and electronic scale. The mean density of PMMA is $1166.14 \mathrm{~kg} / \mathrm{m}^{3}$ with maximum error value of $0.06 \%$. Refer to the national standard of GBT9341-2008, the Elasticity modulus of PMMA is measured by HD-16A static resistance strain gauge and sensor through being loaded step-by-step. The five test specimen is loaded with a constant rate to measure strainstress curve when the material temperature is $20^{\circ} \mathrm{C}, 30^{\circ} \mathrm{C}, 38^{\circ} \mathrm{C}$. The elasticity modulus of PMMA is computed in different temperature and stress. Because of nonlinearity of strain-stress curve in loading initial phase, the PMMA must be preloaded with $0 \leq \sigma_{0} \leq 5 \times 10^{-4} \times \mathrm{E}_{f}$ before measurement, where $\mathrm{E}_{f}$ presents elasticity modulus of static bending. Considering the measured strain-stress relation, stress state of model-bridge and testing fundamental frequency, the elasticity modulus of PMMA is defined as 2.795Mpa.

\section{d) Added weight of model-bridge}

The test material density cannot meet with the similarity scale which can add weight to be satisfied. The additional weight can be computed according to Eq.5. Assuring the stress state of model-bridge and making model-bridge expediently in testing, the model-bridge is added weight through punching in the web of $\mathrm{T}$ beam. The added weight block is fixed on the web with bolt. There are $285 \mathrm{~kg}$ added weight blocks which have been distributed on 170 points all the model-bridge. The similarity constants of the scale-model are computed according to the measured constant of model test-bridge and the similarity scale of Eq. 1 to Eq. 5.

\section{3) Vehicle model}

Vehicle structure is composed of many components such as power system, drive system, tires and wheels, frame and body of vehicle, steering system, braking system and so on. There are many factors which affect the dynamic response of bridge subjected to running vehicle according to driving dynamic characteristic of vehicle, liking weight distribution of vehicle, suspension stiffness of vehicle, wheel stiffness, vehicle frame and vehicle body. Taking three-axle truck as prototype vehicle, the model car is made based on the similarity relation of Eq. 6 . The parameters of vehicle are shown in Tab.1.

The suspension of vehicle ensures that the axle is connected with the frame elastically, which has many functions, for instance, load transfer, releasing impact, retarding vibration and changing the position of running vehicle body. Stiffness of wheel larger than stiffness of suspension greatly, while the proportion of wheel quality in the whole body only occupies $7.4 \%$. The dynamic characteristic of model car suspension is produced with six customize compression springs and damper based on the wheels and the suspension springs equivalent to a set of spring in series. Rigid plastic wheels with $\phi 120 \mathrm{~mm} \times$ $25 \mathrm{~mm}$ are chosen as the wheel of model car, which are linked with bolt bar to form axle. Angle iron is used to make vehicle body in terms of geometric scale. Because stiffness of model car's frame larger than suspension greatly, the bending deflection of model car's body has been neglected in the model test. The key question of making model car is the connection of different components of vehicle. Cylinder is welded on the axle following the spring of suspension placed in it. The transverse and longitudinal displacements between the axle and the frame are assured through welding two smooth fine steel bars outside of cylinder. The two steel bars across the hole site which punching in the model car frame. The model car is added weight with steel plate to assure that the gravity center of added weight model car in accordance with theoretical gravity center of vehicle. The scheme of additional weight is designed based on the similarity relation of frequency, stiffness and axle load between model car and actual vehicle.

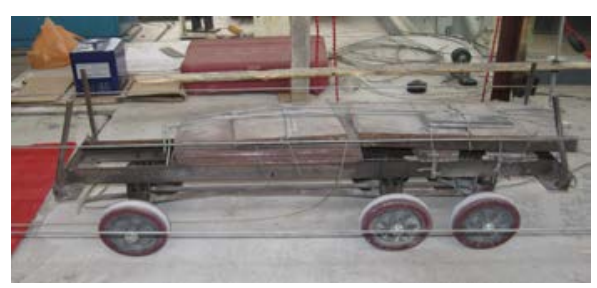

Fig. 3. Model car

\section{4) Model system of traction, braking and limiting}

The model test car is pulled by wire rope winded around the roller, which the roller is drawn by variable speed motor. The fixed pulley is set on the end of bridge to assure wire rope drawn with fixed direction. Considering the characteristic matching of traction system, 3kW variable-frequency motor and ADS-A 5/3 30030 frequency converter are chosen to pull model car. The motor is connected with roller which is similar 
to the working principle of winch. The connection type of motor and roller is shown in Fig.4.

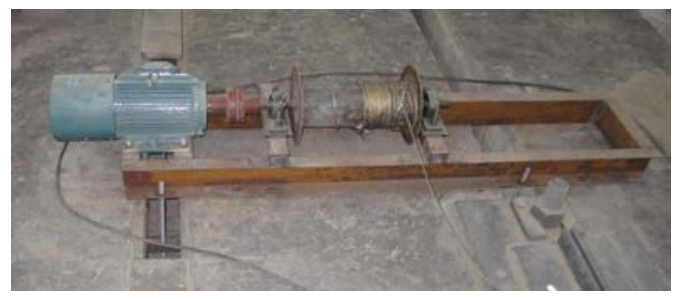

Fig. 4. Frequency conversion motor and expansion cylinder

A set of mechanical equipment is set on the deceleration region of leaving bridge to shut off power when model car across the equipment. The model car can stop quickly depending on reverse tension caused by the sandbag behind the model car. Two sets series springs is set between the tailstock and sandbag to reduce the damage of model car and modelbridge, at the same time, foam-rubber cushion and carpet are laid on the deceleration region.

In order to insure the car straight running on normal lane, the way is designed as follow. Firstly, steel bracket is installed on each pier between bridge approach and leaving bridge. Then guide steel wire ropes are fixed on the screw through punching in the steel bracket. Four large diameter rebar is welt on the frame of test car to limit the transverse movement. The lateral movement of model car running route is controlled by steel tube with strong flexural stiffness in testing region of modelbridge. The limiting equipment insures that the model car runs on the bridge with the same route every time. The whole layout of model test system is shown in Fig.5.

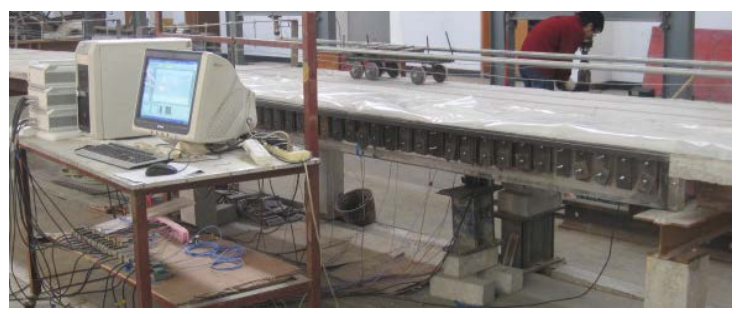

Fig. 5. Equipment of scale-model test

5) Layout of measure point and acquisition system of data

The responses of strain, displacement and velocity are researched in some points liking mid-span, quarter point of bridge and three-quarter point of bridge. Two strain gages are stick under the U-shape bottom of $\mathrm{T}$ beam at each point where the mid-span of each beam and L/4 and 3L/4 of beam 1 to beam 3. There have 22 strain gages all the bridge. Eddycurrent sensor and dial indicator are placed under the bottom of each $\mathrm{T}$ beam to measure the displacement. There have two velocity sensors placed on the non-roadway at mid-span. The dynamic responses of strain, displacement and velocity are selected by DH5920 acquisition system. The dial indicator and HD-16A resistance strain gauge are used to measure the static displacement and static strain.

\section{VERIFICATION OF SCALE-MODEL TEST EQUIPMENT}

\section{A. Numerical Model of Vehicle-Bridge Coupling System}

1) Bride model of mass-spring-damper system

The response of vehicle-bridge coupling system is resolved with MATLAB while the vehicle is simplified as nine-free degree spring-mass-damp system which is similar to reference [15]. The simple supported beam bridge is modeled as shell element and solid element to build the finite element model. The dynamic characteristics including frequency and mode have been extracted by means of ANSYS. The $\mathrm{C}_{50}$ cast-in-site concrete of bridge deck pavement and diaphragm have been modeled as shell63 element, the solsh190 element is used in beam structure. Crash barrier which can be regarded as mass unit placed on the side beam. Neglecting the effect of bituminous concrete on bridge stiffness, the bituminous concrete is modeled as mass 21 element laying all the bridge deck. An equivalent stiffness of beam section is adopted to consider effect of reinforcing steel bar on building finite element model. Bridge finite element model is shown in Fig.6.

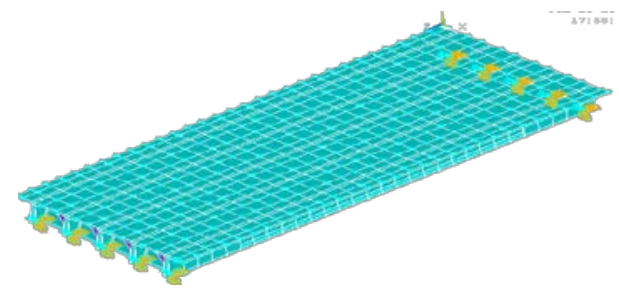

Fig. 6. Bridge finite element model using ANSYS

\section{2) Bridge model of LS-DYNA simulation}

The effects of bridge deck pavement, crash barrier and diaphragm plate on the dynamic characteristic of bridge are considered in establishing the finite element model of bridge using LS-DYNA. Different type of solid element with 8-node or 6-node solid is used in building the finite element model according to the structural characteristic of bridge. The selection of element size and element type depended on computing time and the stability of contact algorithm in solving vehicle-bridge coupling system. In the 3D finite element model of bridge, bridge end transition slab is set in the front of bridge, breaking joint is applied to simulate the expansion joint between two span simple supported girder with continuous deck-pavement. The detailed method of building model using LS-DYNA refers to reference [8]. The numerical visualized model of vehicle-bridge coupling vibration with LSDYNA is shown in Fig.7.

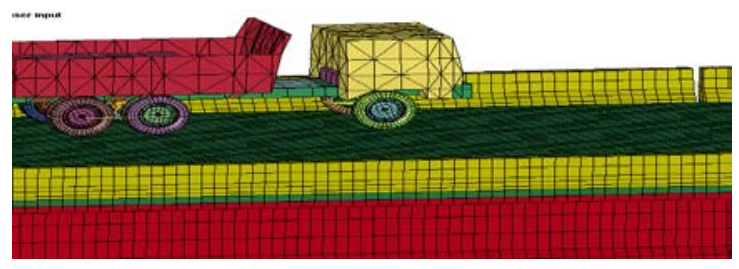

Fig. 7. Numerical visualized model of vehicle-bridge coupling using LSDYNA 


\section{B. Comparison of Dynamic Characteristic}

\section{1) Dynamic characteristic of bridge}

The theoretical frequency of actual bridge is $3.768 \mathrm{~Hz}$ utilizing ANSYS to build finite element model and implement modal analysis. The calculative frequency of model-bridge is $11.915 \mathrm{~Hz}$ according to the frequency similarity relation $\lambda_{f}=\lambda_{l}{ }^{1 / 2}$ when geometric scale is equal to 10 . The PMMA with elasticity modulus $\mathrm{E}=2.795 \times 10^{4} \mathrm{MPa}$ is applied to make model test bridge, and the theoretical frequency of model bridge added weight is $11.924 \mathrm{~Hz}$ in the light of similarity law. The measured frequency of model test bridge added weight is $12.5 \mathrm{~Hz}$, which has error value $4 \%$ compared with theoretical frequency. The error between measured frequency and theoretical frequency comes from the rough end constraint of model test-bridge which induces the elasticity modulus increase and measured frequency larger than theoretical frequency. Considering the effect of continuous deck-pavement on dynamic characteristic, the theoretical frequency of simple support girder-bridge is $3.876 \mathrm{~Hz}$ using LS-DYNA while just with $2.8 \%$ error relative to ANSYS.

\section{2) Dynamic characteristic of vehicle}

The calculative frequency of spring-mass vehicle model is $1.68 \mathrm{~Hz}$ according to the parameter of Tab.1, while the theoretical frequency of vehicle is $1.634 \mathrm{~Hz}$ using LS-DNYA to build the visualized numerical model. The error is $2.7 \%$ between the before-mentioned two methods. The measured frequency of model car is $5.45 \mathrm{~Hz}$ through exerting excitation on the model car, while the theoretical frequency of model car is $5.3 \mathrm{~Hz}$ in terms of frequency similarity relation. The frequency error of model car is $2.8 \%$ between theoretical value and measured value. The measured frequency of model car is in agreement with the theoretical frequency. The dynamic property of making model car can meet with the dynamic characteristic requirement of model test based on the analysis of vehicle frequency.

\section{Comparison of Dynamic Response of Vehicle-Bridge Coupling System}

The displacement and velocity responses of bridge have been researched in order to checkout the feasibility of model scale equipment of vehicle-bridge coupling system. The dynamic displacement of each beam is resolved by numerical simulation of spring-mass-damper system, simulation analysis with LS-DYNA and measuring in model test when a vehicle running on the smooth bridge deck as Fig.1, while the static displacement and strain depend on measuring in test and numerical calculation with ANSYS. In this paper, the model car takes the speed of $6 \mathrm{~m} / \mathrm{s}$ as an example to research the response of vehicle-bridge coupling vibration which is equal to $18.9 \mathrm{~m} / \mathrm{s}$ in actual vehicle.
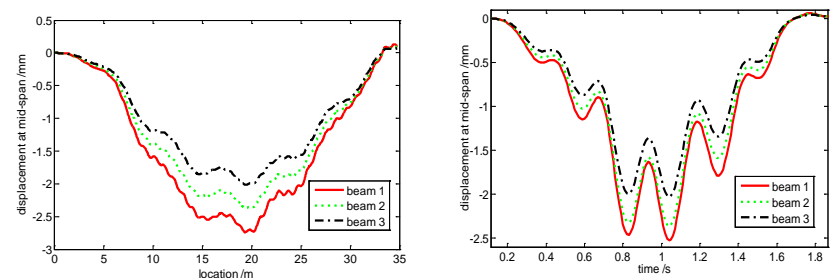

(a)Numerical simulation of mass-spring-damper system analysis with LS-DYNA

Fig. 8. Dynamic deflection response of practical bridge with numerical analysis at span centre
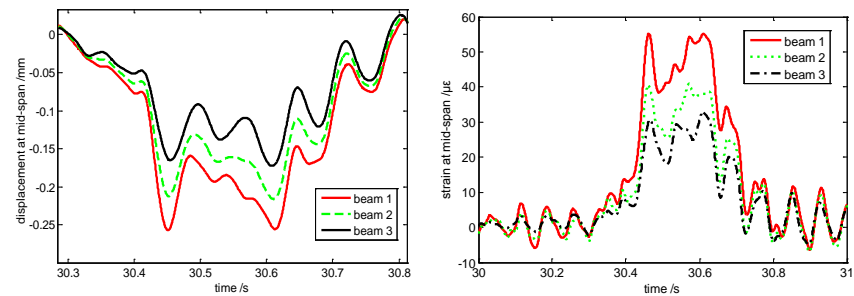

(a)Response of displacement

(b) Response of strain

Fig. 9. Measured dynamic response of model-bridge at mid-span

The displacement response of beam 1 to beam 3 at the midspan is resolved through numerical simulation of spring-massdamper system and simulation analysis with LS-DYNA as shown in Fig.8. The measured dynamic displacement and strain curve of model-bridge at mid-span is shown in Fig.9. The measured displacement response of model-bridge is one-tenth of prototype-bridge in terms of Fig.8 to Fig.9. There emerges the identical peak of dynamic response using above-mentioned three methods. Among these responses, vibration curve of simulation analysis with LS-DYNA is closest to the measured results of model-bridge, while the response of numerical simulation with spring-mass-damper system has more smoother curve than simulation analysis with LS-DYNA and experimental measurement. These results can be ascribed to the simplified vehicle model and neglecting the influence of car body flexibility in spring-mass-damper system.

The comparison of dynamic response among numerical simulation of spring-mass-damper system, visual analysis with LS-DYNA and scale-model test is shown in Table II. At the same time, some conclusions can be obtained as follow. 
TABLE II. THE COMPARISON OF DYNAMIC RESPONSE AT BRIDGE MIDPOINT USING THREE DIFFERENCE METHODS

\begin{tabular}{|c|c|c|c|c|}
\hline \multicolumn{2}{|c|}{ Mechanics index } & 1 & 2 & 3 \\
\hline \multirow{3}{*}{$\begin{array}{c}\text { Max static } \\
\text { displacement } \\
(\mathrm{mm})\end{array}$} & Model test & 0.255 & 0.215 & 0.178 \\
\hline & result of MATLAB & 2.504 & 2.192 & 1.835 \\
\hline & result of LS-DYNA & 2.315 & 2.145 & 1.935 \\
\hline \multirow{3}{*}{$\begin{array}{c}\text { Max dynamic } \\
\text { displacement } \\
(\mathrm{mm})\end{array}$} & Model test & 0.279 & 0.235 & 0.188 \\
\hline & result of MATLAB & 2.741 & 2.374 & 2.016 \\
\hline & result of LS-DYNA & 2.526 & 2.363 & 2.030 \\
\hline \multirow{3}{*}{ Displacement IM } & Model test & 0.094 & 0.093 & 0.086 \\
\hline & result of MATLAB & 0.095 & 0.083 & 0.099 \\
\hline & result of LS-DYNA & 0.091 & 0.102 & 0.049 \\
\hline \multirow{3}{*}{$\begin{array}{l}\text { Maximum static } \\
\text { strain }(\mu \varepsilon)\end{array}$} & Model test & 50.45 & 36.78 & 27.96 \\
\hline & result of MATLAB & 47 & 39.4 & 32.1 \\
\hline & result of LS-DYNA & 43.82 & 35.24 & 28.91 \\
\hline $\begin{array}{c}\text { Max dynamic } \\
\text { strain }(\mu \varepsilon)\end{array}$ & Model test & 55.22 & 40.46 & 30.7 \\
\hline IM of strain & Model test & 0.095 & 0.100 & 0.098 \\
\hline
\end{tabular}

(1)The error is defined as the difference value of theoretical value and measured value to theoretical value ratio. Among all measured points, the static displacement and static strain of side beam generate maximum error. The maximum error of displacement is $2.8 \%$ and the maximum error of strain reaches $3.1 \%$.

(2)The variation trend of model-bridge measured static displacement and static strain is in accordance with theoretical value of prototype-bridge, while the measured displacement and strain of model-bridge has more obvious lateral extent of variation than theoretical value of actual bridge. The results can be attributed to equivalent thickness of bridge deck which induces larger stiffness than actual bridge.

(3)The peak of measured dynamic displacement of modelbridge is close to spring-mass-damper system, while the result of simulation analysis using LS-DYNA is relatively small. The main reason is that bridge deck pavement participates in force in the model of LS-DYNA which results in smaller response than model test and spring-mass-damper system.

(4)The impact coefficients of displacement and strain have the same variation law using three above-mentioned methods, which range from 0.08 to 0.10 . Because of larger similarity scale of strain and displacement in designing model test, the weaker error of measured displacement and strain will induce large error of impact coefficient. In order to improve the testing accuracy and reduce the error of impact coefficient, the small similarity scale of strain and displacement can be used in the future experiment design.

\section{CONCLUSION}

According to the characteristics of highway bridge-vehicle coupling vibration, the scale model test equipment of vehiclebridge coupling vibration has been designed and manufactured. The effectiveness of the dynamic characteristic of model car and model-bridge and the dynamic response of scale model system of vehicle bridge coupling has been checked according to the measured results and numerical results. The research results show the conclusion as follow.
(1)The measured frequency of model test-bridge has error value $4 \%$ compared with theoretical frequency, the maximum error of displacement is $2.8 \%$ and the maximum error of strain reaches $3.1 \%$. The frequency error of model car is $2.8 \%$ between theoretical value and measured value.

(2)There have larger error in the third, fourth and sixth frequency of model bridge caused by the stiffness of modelbridge larger than actual bridge.

(3) The impact coefficients of displacement and strain have the same variation law between the model-bridge and actual bridge using three different methods.

(4)The effect of temperature on vibration response should be paid more attention during test procedures because of the characteristic of PMMA. In order to improve the test precision, the similarity scales of strain and displacement can be enlarged in future test.

\section{ACKNOWLEDGEMENTS}

The authors acknowledge the financial support provided by the Natural Science Foundation China (Project Nos. 51268013, 51468018)

\section{REFERENCES}

[1] Song Yi-fan. "Gonglu Qiaoliang Dongli Xue,” BeiJing, China Communications Press, 2000.(In Chinese)

[2] K.Henchi, M.Fafard. "An Efficient Algorithm for Dynamic Analysis of Bridges under Moving Vehicles Using a Coupled Modal and Physical Components Approach,” Journal of Sound and Vibration, Vol. 212, pp. 663-683,Appil 1998.

[3] Hong-yi Li. "Dynamic Response of Highway Bridges Subjected to Heavy Vehicles. Florida State University Famu-fsu college of engineering," 2005.

[4] Dongzhou Huang. "Dynamic and Impact Behavior of Half-Through Arch Bridges. Journal of Bridge Engineering,"Vol.10, pp.133-141, February,2005.

[5] Magdy Samaan, John B.Kennedy, Khaled Sennah. Impact Factors for Curved Continuous Composite Multiple-Box Girder Bridges. Journal of Bridge Engineering, Vol.12, pp.80-88, January,2007.

[6] Hongyi Li, Jerry Wekezer, Leslaw Kwasniewski. “Dynamic Response of a Highway Bridge Subjected to Moving Vehicles,” Journal of Bridge Engineering, Vol.13, pp.439-448, May, 2008.

[7] Zhang Nan, Xia He. "Dynamic Analysis of Railway Bridge under high speed train,” Engineering Mechanics,Vol. 22, pp.144-151,March, 2005. (In Chinese)

[8] Gui Shui-rong, Liu Lv, Chen Shui-sheng, Zhao Hui. "Research on Models of a Highway Bridge Subjected to a Moving Vehicle Based on the LS-DYNA Simulator," Journal of Highway and Transportation Research and Development, Vol.8,pp.76-82, September, 2014. (In Chinese)

[9] Xiang Yi-qiang, Wu Sun-yao, Duan Yuan-feng. "Design Method of Small-scale Cable-stayed Bridge Based on Stiffness Similarity Theory,” Journal of Experimental Mechanics, Vol, 25, pp.438-444, April, 2010.

[10] Wang Shu-guang, Liu Wei-qing, Xu Xiu-li, Zhong Dong-qing. “Shaking Table Model Test of Long-span Continuous Girder Bridge with Longitudinal Seismic Energy Dissipating,” China Journal of Highway and Transport, Vol.22, pp.54-59, October, 2009. (In Chinese)

[11] Fan Ke, Li Zhen-bao, Yan Wei-ming. "Vibration table tests for the archbridge under multi points inputs,” Journal of railway science and engineering, Vo.4, pp.19-24, December, 2007.

[12] Tommy H.T. Chan, Demeke B.Ashebo. "Moving Axle Load From Multi-Span Continuous Bridge: Laboratory Study,” Journal of vibration and Acoustics, Vol,128, pp.521-526, September,2006. 
[13] Chi Shi-chun, LAM siu-shu. "Validation of similitude laws for dynamic structural model test,”World earthquake engineering,Vol.20, pp.11-20 August, 2004. (In Chinese)

[14] Shui-rong GUI, Shuisheng Chen. "Yongming Ren. Study on vibration test of the scale-model system for highway bridge-vehicle interaction according to the similarity law," The international conference on remote sensing environment and transportation engineering, 2011. (In Chinese)
[15] Gui Shuirong, Chen Shuisheng, Ren Yongming. "Research on Influencing Factors of Impact Coefficient of Continuous Beam Bridge Transformed form Simply-Supported Structure Subjected to Moving Vehicle Loads,” Journal of Highway and Transportation,2011. (In Chinese) 